\title{
Experimental Study of Surface and Solution Properties of Gemini -conventional Surfactant Mixtures on Solubilization of Polycyclic Aromatic Hydrocarbon
}

\author{
M. Kamil", Huma Siddiqui \\ Department of Petroleum Studies, Aligarh Muslim University, Aligarh-202002, India \\ Email: *sm_kamil@rediffmail.com
}

Received May, 2013

\begin{abstract}
Experimental data are presented on the enhanced solubilities of fluorene (FLR) resulting from solubilization in aqueous solutions of two conventional surfactants: cationic cetyltrimethylammonium bromide (CTAB) , anionic sodium dodecyl sulfate (SDS), nonioinic polyethylene glycol dodecyl ether (Brij35) and a cationic gemini bis (hexadecyldimethylammonium) pentane dibromide (G5). The critical micellar concentration of surfactants was determined by surface tension measurements and aqueous solubilities of fluorene compound in surfactant solutions were measured spectrophotometrically. Solubilization of PAH compound commenced at the surfactant critical micelle concentration and was proportional to the concentration of surfactant in micelle. The results of the mixed systems were analyzed with the help of regular solution theory, in which the deviation of $\mathrm{CMC}_{\exp }$ values for mixed surfactant systems from $\mathrm{CMC}_{\text {ideal }}$ was measured by evaluating the interaction parameter, $\beta^{\mathrm{m}}$. Negative values of $\beta^{\mathrm{m}}$ were observed in all equimolar binary systems which show synergism in the mixed micelle. Attraction force between two oppositely charged head groups lead the strongest synergism effect between cationic gemini and anionic conventional surfactant. In addition to molar solubilization ratio (MSR) solubilization efficiency is also quantified in terms of micelle-water partition coefficient $\left(\mathrm{K}_{\mathrm{m}}\right)$.
\end{abstract}

Keywords: Gemini Surfactants; PAHs; Solubilization; Mixed micelle; Solution Properties

\section{Introduction}

Hydrophobic organic contaminants (HOCs) are found in the priority list of hazardous substances as listed by the EPA and the agency for toxic substances and disease registry of USA [1]. The hrdrophobicity of these contaminants is one of the factors that determine the fate of the contaminant in the environment. Contamination of soil and underground water by persistent organic pollutant is a major environmental concern. Polycyclic aromatic hydrocarbons (PAHs) are one class of such pollutants. They are made of two or more fused benzene rings formed mainly from the combustion of fossil fuels and are always found as a mixture of individual compounds. They are of special interest because (i) they are known or suspected carcinogens or mutagens and (ii) they strongly adsorb to soil sediments making them persist in the soil for extended periods of time [2-7]. As a consequence, remediation of PAHs in soil-water system is often dependent on desorption of the contaminant from the soil surface and its subsequent incorporation into the bulk aqueous phase. In addition, owing to their low aqueous

${ }^{*}$ Corresponding author. solubility and low vapor pressure, their removal from the environment water and soil still presents a considerable challenge to researchers involved in separation technology. One promising technique, surfactant enhanced subsurface remediation (SESR) has emerged in which the solubility of organic solutes is greatly enhanced by the presence of surfactant micelles [8-13]. Micelles are self-association of surfactant molecules with the surfactant hydrophobic portion oriented towards the center of the aggregates and the hydrophilic portions located at the aggregate surface and facing the solvent molecules. The central core of the micelle thus constitutes a hydrophobic pseudo phase that may accommodate a certain amount of a lipophilic solubilizate, resulting in an enhancement of its solubilization. To get a better system, mixed micellar systems have already been used for the significant enhancement of water solubility of poorly soluble organic contaminants. Mixed surfactants improve the performance of surfactant -enhanced remediation of soils and sediments by decreasing the applied surfactant level and thus its cost.

Conventional surfactant molecules are composed of a long hydrophobic hydrocarbon tail with an ionic or polar 
hydrophilic head. Gemini surfactants are made up of two hydrocarbon tails and two ionic head groups connected by a 'spacer' in the sequence: hydrocarbon tail / ionic group / spacer / ionic group / hydrocarbon tail [14]. The spacer can be attached directly to the identical ionic groups, each of which is in turn bonded to an identical hydrocarbon tail; alternatively, the two identical amphiphiles are joined midway. Their various surface active properties are superior to those of corresponding conventional surfactants with one hydrophilic and one hydrophobic group. Thus, they have much lower CMC values and are more efficient in lowering the surface tension of water. As a result, the Gemini surfactants form larger micelles than the conventional surfactants and thus should have a better solubilizing capacity [15-16].

Thus in the open literature, only few studies are reported on solubilization of PAHs in Gemini-conventional mixed surfactant systems are available. The objectives of the present study are: (i) to compare the efficiency of few Gemini-conventional mixed surfactants in enhancing the water solubility of Fluorene and (ii) to have the idea about the synergistic solubilization of Fluorene by mixed suefactant systems. This experimental study is aimed to ascertain if a mixed surfactant solution may be used in the SER of organic pollutants. In this study, we have studied interfacial properties and molecular interactions of individual as well as equimolar binary mixtures of cationic gemini and conventional surfactants.

\section{Experimental}

\subsection{Materials Used}

The surfactants cetyltrimethyl ammonium bromide (CTAB), sodium dodecyl sulphate or sodium lauryl sulphate (SDS), and polyethylene glycol dodecyl ether (Brij 35) were purchased from Sigma Aldrich and used as re- ceived. Gemini surfactant pentanediyl-1,5-bis (dimethylcetylammonium bromide), abbreviated as G5 was synthesized in the research laboratory of the Department of Chemistry, AMU, Aligarh. Synthesis of gemini's entail refluxing of 1,6-dibromohexane with $\mathrm{N}, \mathrm{N}$ - dimethylcetylamine (molar ratio 1:2.1) in dry ethanol. For maximum bisquaternization continuous stirring at $80^{\circ} \mathrm{C}$ is done for $48 \mathrm{~h}$ to ensure as much as possible a complete bisquaternization. The progress of the reaction was monitored by using TLC technique. The solvent was removed under vacuum after the completion of the reaction.

Fluorene was used as polycyclic aromatic hydrocarbon in the present work and was also procured by sigma Aldrich chemical company. Surfactant solutions were prepared in double distilled water. Structures of above chemicals are shown in Figure 1.

\subsection{Methods}

\subsubsection{Critical Micelle Concentration Determination by Surface Tension Measurements}

For CMC determination tensiometric experiments were performed for single as well as for mixed surfactant systems. The apparatus used for the purpose was Hardson tensiometer (Hardson make Kolkata, India), which works on ring detachment method. The vertically hung ring was initially dipped in the surfactant solution to measure its surface tension. It was then forced to pull out from the solution. The required force which was applied to pull out the ring from the solution is its surface tension. Experiments were repeated twice for each surfactant to ensure the reproducibility of the results. The surface tension versus log [surfactant] plots for individual and mixed surfactant systems are shown in Figures 2 and, 3 respectively. The concentration at which inflexion in curve is obtained is the CMC of that substance.
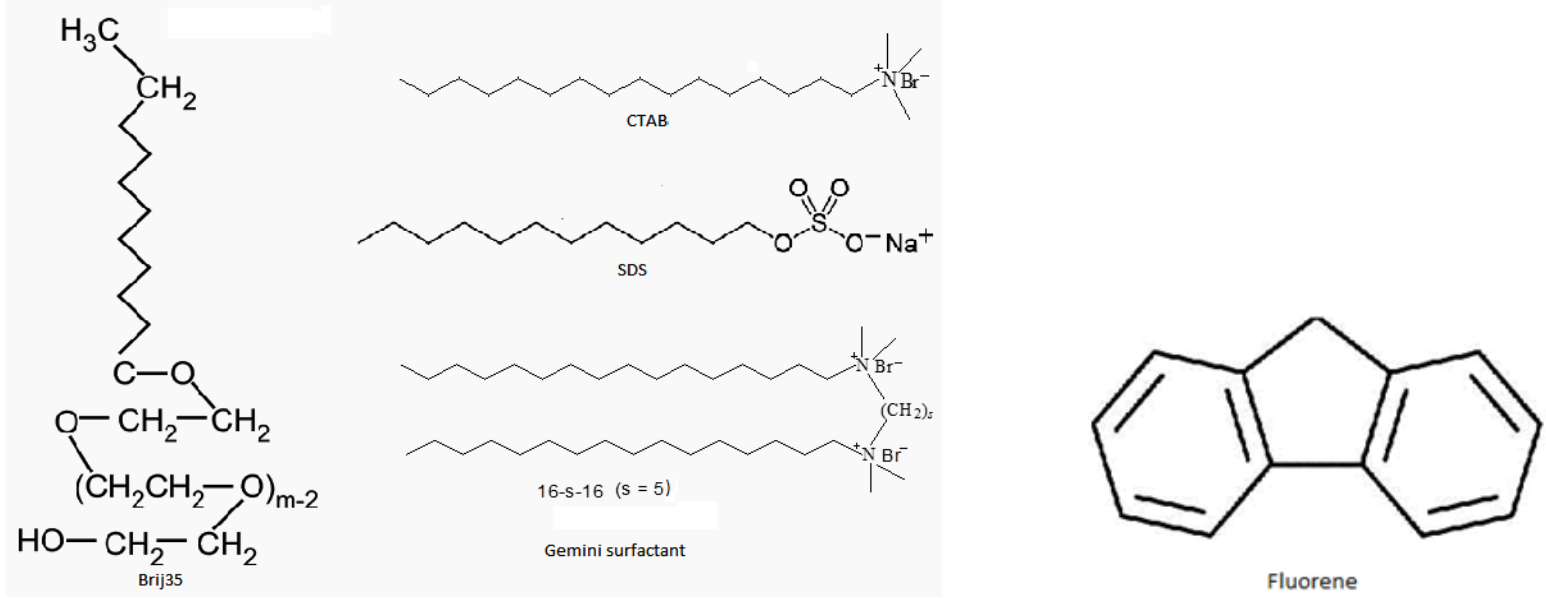

Figure 1. Structures of reagents used in the study polyethylene glycol dodecyl ether (Brij 35), cetyltrimethylammonium bromide (CTAB), sodium lauryl sulphate (SDS), gemini surfactant (G5), and fluorene. 

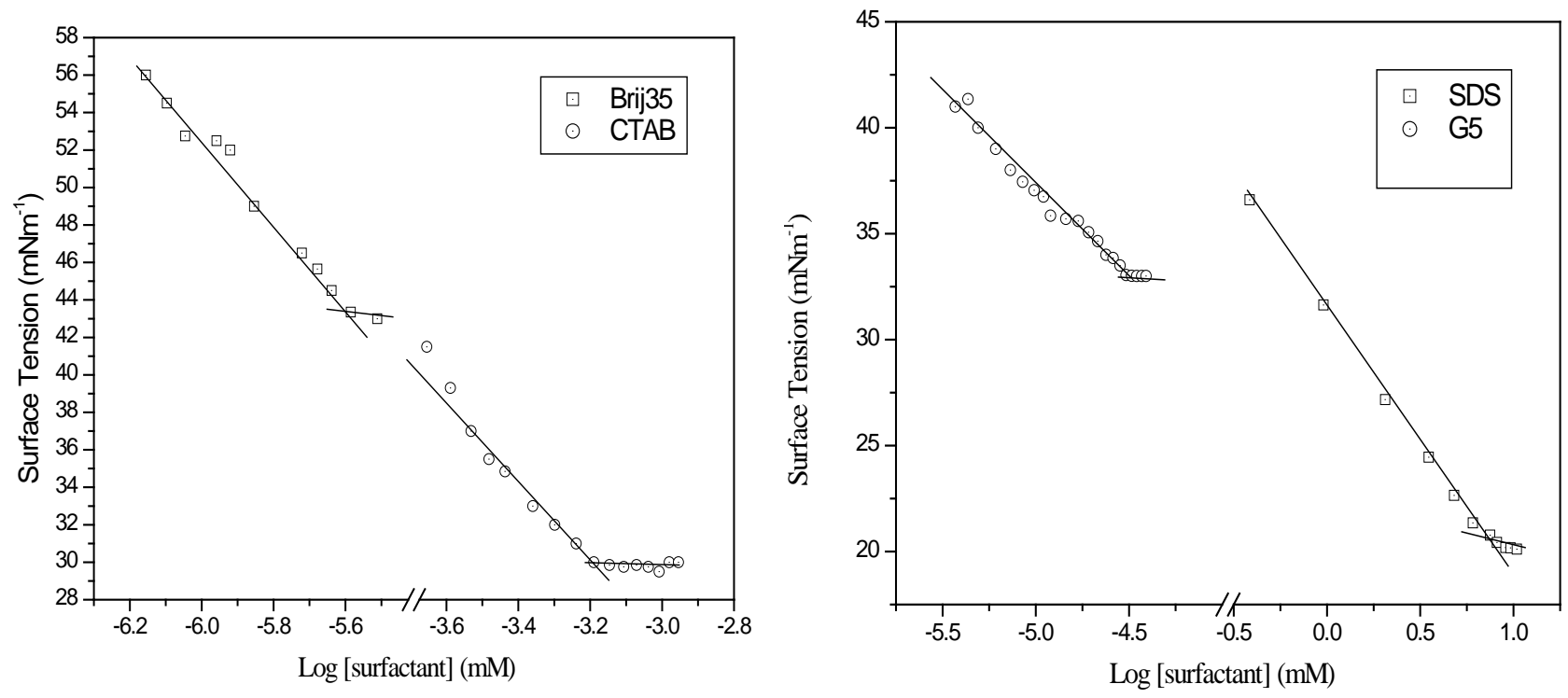

Figure 2. Surface tension versus. log [surfactant] plots for single surfactant systems.

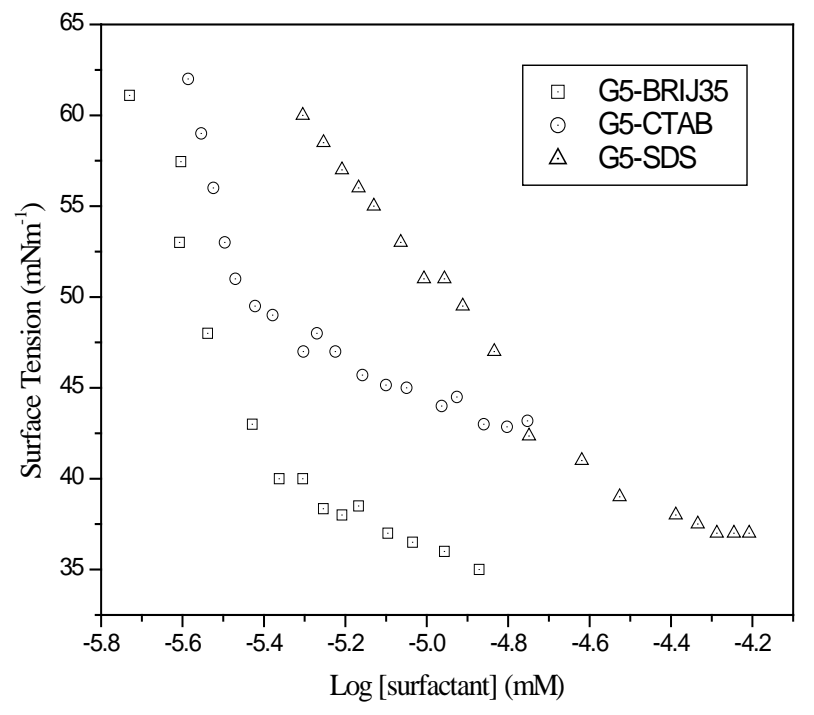

Figure 3. Surface tension versus log [surfactant] plots for G5/conventional mixed surfactant systems.

\subsubsection{Solubilization Experiments}

Solubility of Fluorene in surfactant system was determined by solubilization experiments. Solutions of concentration higher than their corresponding CMC were prepared. These solutions were then filled in borosilicate screw-capped glass vials of capacity of $5 \mathrm{ml}$ with an excess amount of Fluorene. Extra amount of Fluorine was added to ensure maximum solubility in surfactant solution. These samples are then agitated on magnetic stirrer for a period of $24 \mathrm{~h}$ at $30^{\circ} \mathrm{C}$. To ensure good agitation magnetic teflon pieces were also dropped in each vial. After this, a portion of the samples are collected in eppendorf tubes and centrifuged at $12000 \mathrm{rpm}$, using a high speed micro centrifuge (REMI, RM-12C) to settle down the undissolved Fluorene. The concentration of the solubilized Fluorine of centrifuged sample was determined spectrophotometrically using Shimadzu spectrophotometer (model UV mini-1240), following by appropriate dilution of a sample of the supernatant with the corresponding surfactant solution. Before taking spectra baseline correction was done with the surfactant solution of same concentration.

\section{Results and Discussion}

\subsection{Critical Micelle Concentration}

The surfactant concentration at which monomers begin to assemble in ordered, colloidal aggregates or micelle is termed as critical micelle concentration (CMC or $\mathrm{cmc}$ ). The CMC values of pure as well as of binary surfactant mixtures $\left(\mathrm{cmc}_{\text {exp }}\right)$ were evaluated on the basis of tensiometric measurements. Surface tension decreases as the concentration of the surfactant increases. Surfactant molecules at low concentrations adsorb at the liquid/air interface until the surface of the solution is completely occupied. Then the excess molecules tend to self-associ- ate in the solution to form micelles, and surface tension becomes constant. Two opposite effects control micellization: the effect of the hydrophobic group is an important driving force in micellization and the effect of the hydrophilic group opposing it. The cmc values were determined by noting inflections in the surface tension $(\gamma)$ versus logarithm of surfactant concentration isotherms and are given in Table 1. The gemini surfactant has remarkably low cmc value as compared to the conventional surfactants because of its two polar head groups and two hydrophobic chains which transfer at the same time from the aqueous phase to micellar phase. 


\subsection{Interfacial Properties}

The surface properties such as $\Gamma_{\max }$ (the maximum surface excess), $\mathrm{A}_{\min }$ (the minimum surface area per molecule), and thermodynamic parameters, $\Delta G_{m}$ (Gibbs free energy of micellization), $\Delta \mathrm{G}_{a d}$ (the standard Gibbs energy of adsorption, $G_{\min }$ (the free energy of air/water interface) of individual as well as equimolar binary surfactant systems were determined.

The adsorption efficacy of selected surfactants and their mixtures at the air/solution interface were evaluated with the help of the Gibbs adsorption equation [17-19].

$$
\Gamma_{\max }=\frac{1}{2.303 * n * R * T}\left[\frac{d \gamma}{d \log \log X}\right]_{\Gamma, P}
$$

where, $\gamma$ is surface tension of the solution, $\mathrm{R}$ is the universal gas constant $\left(8.314 \mathrm{JK}^{-1} \mathrm{~mol}^{-1}\right)$, $\mathrm{T}$ is temperature in the absolute scale, $\mathrm{X}$ is concentration of the surfactant in solution, and ' $n$ ' is a constant, which depends on the number of species constituting the surfactant.
The factor $\frac{d \gamma}{d \log \log X}$ was obtained from the slopes of the plots of surface tension vs. $\log$ [surfactant]. $\Gamma_{\max }$ values were used to calculate the minimum area per surfactant molecule $\left(A_{\min }\right)$ at the air/water interface using the equation:

$$
A_{\min }=\frac{1}{N^{*} \Gamma_{\max }}
$$

where, $\mathrm{N}$ = Avogadro's Number

The minimum area per surfactant molecule was found to be minimum for Brij 35, and maximum for SDS as given in Table 2. For the case of gemini surfactants, an increase in $A_{\text {min }}$ was observed with increase in carbon number of spacer group. The values of the surface pressure at the CMC $\left(\Pi_{\mathrm{CMC}}\right)$ were obtained from the following equation:

$$
\Pi_{\mathrm{CMC}}=\gamma_{\mathrm{o}}-\gamma_{\mathrm{CMC}}
$$

Table 1. Experimental and literature CMC values of surfactants.

\begin{tabular}{clccc}
\hline S.No. & Surfactant System & $\mathbf{C M C}_{\exp }(\mathbf{m M})$ & $\mathbf{C M C}_{\text {lit }}(\mathbf{m M})$ & $\mathbf{C M C}_{\text {ideal }}(\mathbf{m M})$ \\
\hline 1. & CTAB & 0.67600 & $0.776(24)$ & - \\
2. & Brij35 & 0.00363 & $0.030(65)$ & - \\
3. & SDS & 7.92000 & $8.000(37)$ & - \\
4. & G5 & 0.03160 & $0.036(38)$ & - \\
5. & G5-CTAB & 0.01380 & - & 0.03018 \\
6. & G5-Brij35 & 0.00446 & - & 0.00325 \\
7. & CG-SDS & 0.02750 & - & 0.03147 \\
\hline
\end{tabular}

Table 2. Maximum surface excess $\left(\Gamma_{\max }\right)$, the minimum surface area per molecule $\left(A_{\min }\right)$, degree of micellar ionization , the standard Gibbs energy of adsorption $\left(\Delta G_{a d}\right)$, Gibbs free energy of micellization $\left(\Delta G_{m}\right)$, the free energy of air/water interface $\left(G_{\min }\right)$ values.

\begin{tabular}{lcccccc}
\hline $\begin{array}{l}\text { Surfactant } \\
\text { System }\end{array}$ & $\begin{array}{c}\pi_{\mathrm{CMC}} \\
(\mathbf{m N} / \mathbf{m})\end{array}$ & $\begin{array}{c}\Gamma_{\max }{ }^{*} \mathbf{1 0}^{7} \\
\left(\mathbf{m o l} / \mathbf{m}^{2}\right)\end{array}$ & $\begin{array}{c}\mathbf{A}_{\min } \\
\left(\mathbf{m}^{2} / \mathbf{m o l}\right)\end{array}$ & $\begin{array}{c}\Delta \mathbf{G}_{\text {ad }} \\
(\mathbf{K J} / \mathbf{m o l})\end{array}$ & $\begin{array}{c}\Delta G_{m} \\
(\mathbf{K J} / \mathbf{m o l})\end{array}$ & $\begin{array}{c}\Delta G_{\min } \\
(\mathbf{K J} / \mathbf{m o l})\end{array}$ \\
\hline CTAB & 41.2 & 27.62608 & 60.0991 & -30.0113 & -28.5200 & 10.7869 \\
Brij35 & 26.21 & 31.9085 & 52.0331 & -42.5362 & -41.7148 & 13.0968 \\
SDS & 49.79 & 22.9558 & 73.3206 & -24.4867 & -22.3177 & 8.8517 \\
G5 & 35.98 & 13.8091 & 120.2319 & -38.8455 & -36.2400 & 23.9116 \\
G5-CTAB & 27.52 & 7.9760 & 208.1597 & -41.7784 & -38.3281 & 53.8860 \\
G5-Brij35 & 29.15 & 7.5595 & 219.6291 & -45.0651 & -41.2090 & 52.7146 \\
G5-SDS & 32.79 & 12.7730 & 129.9847 & -39.1573 & -36.5902 & 29.9145 \\
\hline
\end{tabular}


where $\gamma_{0}$ is the surface tension of pure solvent and $\gamma_{\text {CMC }}$ is the surface tension at CMC. The standard Gibbs free energy of micellization per mole was estimated using the equation:

$$
\Delta G_{m}=\mathrm{R} * \mathrm{~T} * \ln \mathrm{CMC} * 0.001
$$

The standard free energy of adsorption at air/water interface can be derived from this standard free energy, as:

$$
\Delta G_{a d}=\Delta G_{m}-\left(\frac{\Pi_{C M C}}{\Gamma_{\max }}\right)
$$

Table 2 clearly shows that both $\Delta G_{m}$ and $\Delta G_{a d}$ are negative and their magnitudes showed a somewhat impulsive nature of $\Delta G_{a d}$, which causes surfactant molecules movements toward air/water interface. With this finding, it was revealed that adsorption is primary and micelle formation process is secondary process, during surfactant addition in water. The maximum $\Delta G_{a d}$ is observed for the G5/Brij 35 combination. Further, $A_{\min }$ of conventional surfactants follow the order SDS $>$ CTAB $>$ Brij35 (anionic $>$ cationic $>$ non-ionic). For the mixed systems the order is: G5-Brij35 > G5-CTAB > G5-SDS.

\subsection{Surfactant - surfactant Interaction}

To determine, whether the binary systems follows ideal or nonideal behavior, the experimental CMC values of equimolar binary surfactant systems were compared with ideal CMC values. The $\mathrm{CMC}_{\text {ideal }}$ values were calculated using Clint equation [20]:

$$
\frac{1}{\mathrm{CMC}_{\text {ideal }}}=\frac{\alpha \alpha}{\mathrm{CMC}_{1}}+\frac{2}{\mathrm{CMC}_{2}}
$$

where $\mathrm{CMC}_{1}, \mathrm{CMC}_{2}, \alpha_{1}$ and $\alpha_{2}$ are the critical micelle concentrations and the mole fractions of component 1 and 2 in mixed surfactant solutions. In Table 1, it is observed that all $\mathrm{CMC}_{\exp }$ values were less than $\mathrm{CMC}_{\text {ideal }}$, as predicted by above equation which shows that the formation of mixed micelles exhibits a negative deviation with respect to ideal mixture.

From Table 1, it is clear that CMCs of ionic surfactants are much higher than nonionic surfactant. This fact can be justified as nonionic surfactant molecules show hydrophobic interaction among hydrocarbon chains, which are easily separated from the aqueous environment, whereas ionic surfactants requires higher concentrations to overcome the electrostatic repulsion between ionic head groups while aggregating [21]. Moreover it was also observed that the $\mathrm{CMC}_{\exp }$ values of binary systems are lower than their corresponding ideal values, which indicate synergistic interaction in all mixed systems.

In the light of the regular solution theory, the deviation of $\mathrm{CMC}_{\exp }$ values for mixed surfactant systems from $\mathrm{CMC}_{\text {ideal }}$ can be measured by evaluating the interaction parameter, $\beta$. This parameter can be calculated with the help of Rubingh's equation [22]:

$\beta=\frac{\ln \ln \left(\begin{array}{c}\mathrm{CMC}_{2} \mathrm{CMC}_{\mathrm{H}} / \mathrm{CMC}_{1} \mathrm{X}_{1}^{\mathrm{m}} \\ \mathrm{C}\end{array}\right)}{\left(1-\mathrm{X}_{1}^{\mathrm{m}}\right)^{2}}=\frac{\ln \ln \left({ }^{12} / \mathrm{CMC}_{2} \mathrm{X}_{2}^{\mathrm{m}}\right)}{\left(1-\mathrm{X}_{2}^{\mathrm{m}}\right)^{2}}$

where $\mathrm{X}_{1}^{\mathrm{m}}, \mathrm{X}_{2}^{\mathrm{m}}$ are the micellar mole fraction of surfactant 1 and 2 in the mixed micelles, and $\mathrm{CMC}_{1}, \mathrm{CMC}_{2}$ and $\mathrm{CMC}_{12}$ are critical micelle concentrations of surfactants 1 and 2, and CMC of mixed surfactant system, consisting of surfactant 1 and 2, both respectively, $\alpha_{1}$, and $\alpha_{2}$ are their corresponding bulk mole fractions. The micellar mole fraction $X_{i}^{m}$ was calculated with the help of following equation for nonideal binary mixture of surfactants by solving iteratively [22,23].

$$
\frac{\mathrm{X}_{1}^{\mathrm{m} 2} \ln \ln \left(\frac{\alpha \mathrm{CMC}_{12}}{\mathrm{X}_{1}^{\mathrm{m}}}\right)}{\left(1-\mathrm{X}_{1}^{\mathrm{m} 2}\right) \ln \ln \left[\left(1 \propto \mathrm{CMC} \quad 12 /\left(1-\mathrm{X}_{1}^{\mathrm{m}}\right) \mathrm{CMC}_{12}\right]\right.}=1
$$

A negative value of $\beta$ shows negative deviation of $\mathrm{CMC}_{\text {exp }}$ from $\mathrm{CMC}_{\text {ideal, }}$ which indicates a reduction in free energy of micellization over that predicted by the ideal solution theory [23]. This implies good interaction between surfactants in mixed system. A positive value of $\beta$ signifies antagonism between components of surfactant combination. Another parameter, activity coefficient $f_{1}$ and $f_{2}$ within the mixed micelles derived from Rubingh equations was equated as :

$$
\begin{aligned}
& f_{1}=\exp \exp \left\{1\left(-X^{m}\right)^{2}\right\} \\
& f_{2}=\exp \exp \left\{\left(X^{m}\right)^{2}\right\}
\end{aligned}
$$

The values of $\beta$ obtained experimentally for the selected surfactants are given below in Table 3 .

All negative $\beta$ values in Table 3 indicate good interaction between the components of mixed system and demonstrate synergistic effect for all binary equimolar mixed surfactant systems. The larger negative value of $\beta$ denotes the greater negative deviation of $\mathrm{CMC}_{\exp }$ from $\mathrm{CMC}_{\text {ideal }}$. The order of deviation exhibited through $\beta$ is G5-SDS > G5-CTAB > G5-Brij35. The strongest synergism effect is found between cationic gemini and anionic conventional surfactant. The reason behind this might be the attractive forces between oppositely charged head groups. The least value was for cationic gemini and nonionic conventional surfactant, as Brij35 has polyoxyethylene (POE) groups with large number of oxygen atoms and a lone pair of electron, thus it may have a tendency to react coulombically with cationic gemini surfactant, but the 
existence of long polyoxyethylene head group imposes some steric constraints due to thermal vibrations, which causes control on effective head group interactions and give reason to reduce the value of $\beta[24,25]$.

Rubingh's model observes the attractive interaction in the mixed micelle formation. To analyze interaction between the amphiphiles in a mixed surfactant system at air/water interface, Rosen model was used. According to Rosen model the mole fraction of surfactant 1 at the mixed adsorbed film can be calculated iteratively as [26]:

$$
\frac{\mathrm{X}^{\sigma^{2}} \ln \ln \left(\frac{\alpha C_{12}}{C_{1} X_{1}^{\sigma}}\right)}{\left(1-X^{\sigma}\right)^{2} \ln \left[\left(1 \Theta C_{1}\right){ }_{12} /\left(1-X_{1}^{\sigma}\right) C_{2}\right]}=1
$$

where $\mathrm{C}_{12}, \mathrm{C}_{1}$ and $\mathrm{C}_{2}$ are the concentration of mixture at a fixed surface tension value and the concentrations of individual surfactants at a fixed surface tension value and $\alpha_{1}$ was the mole fraction of surfactant 1 . From this expression the value of $X^{\sigma}$ was obtained, which was then used to evaluate the interaction parameter, $\beta^{\sigma}$ at air/ water interface, with the help of following equation:

$$
\beta^{\sigma}=\frac{\ln \ln \left(\mathrm{C}_{2} \mathrm{C} \alpha / \mathrm{C}_{1} \mathrm{X}_{1}^{\sigma \sigma}\right)}{\left(1-\mathrm{X}_{1}^{\sigma \sigma}\right)^{2}}=\frac{\ln \ln \left(122 / \mathrm{C}_{2} \mathrm{X}_{2}\right)}{\left(1-\mathrm{X}_{2}\right)^{2}}
$$

The values of $\beta^{\sigma}$ and $X^{\sigma}$ are presented in Table 4 . The negative values of $\beta^{\sigma}$ indicate attractive interaction. The activity coefficients ( $f_{1}^{\sigma}$ and $f_{2}^{\sigma}$ ) were calculated through Rosen approach within the mixed micelle with the help of interaction parameters as given below:

$$
\begin{aligned}
& f_{1}^{\sigma}=\exp \exp \left\{1^{\sigma}\left(-X^{\sigma}\right)^{2}\right\} \\
& f_{2}^{\sigma}=\exp \exp \left\{{ }^{\sigma}\left(X^{\sigma}\right)^{2}\right\}
\end{aligned}
$$

From Table 4, it was observed that all the systems exhibits positive value of interaction parameter, which shows antagonistic effect between components of mixed surfactant system.

\subsection{Solubilization by Single Surfactants}

To determine the extent of solubilization of PAH in surfactant, absorbance of PAH in surfactant solution (of known concentration) is checked with the help of spectrophotometer. Before examining the solubilization power of binary mixtures, single systems were first studied, to get an idea about the efficiency of gemini in comparison with conventional surfactants. Graphs of the solubility of FLR as a function of the concentration of surfactant are plotted in Figures 4 and 5. Both plots show that on increasing surfactant concentration, concentration of dissolved FLR is also increasing, or its solubility increases linearly with the increasing surfactant concentrations above CMC [27]. This behavior indicates that solubilization is related to micellization. Though, the reduced CMC value does not absolutely represent the increased solubilization ability. Thus, water solubility enhancement of FLR by selected single and equimolar binary surfactant systems was further to evaluate and compare.

Table 3. Micellar mole fraction $\left(X_{1}^{m}\right)$, interaction parameter $(\beta)$, activity coefficients $\left(f_{1}\right.$ and $\left.f_{2}\right)$ values for gemini/conventional mixed surfactant systems at $30^{\circ} \mathrm{C}$

\begin{tabular}{lccccc}
\hline Surfactant System & $\boldsymbol{X}_{1}^{m}$ & $\boldsymbol{\beta}$ & $\boldsymbol{f}_{1}$ & $\boldsymbol{f}_{2}$ & $\begin{array}{c}\Delta \mathrm{G}_{\mathrm{ex}} \\
(\mathbf{K J} / \mathbf{m o l})\end{array}$ \\
\hline G5-CTAB & 0.6475 & -8.37763 & 0.35311 & 0.02983 & -4.81696 \\
G5-Brij35 & 0.2672 & -2.47934 & 0.26411 & 0.83777 & -1.22296 \\
G5-SDS & 0.7515 & -8.84881 & 0.57901 & 0.00676 & -4.16287 \\
\hline
\end{tabular}

Table 4. Surface composition at air/water interface $\left(X_{1}^{\sigma}\right)$, interaction parameter $\left(\beta^{\sigma}\right)$, activity coefficients $\left(f_{1}^{\sigma}\right.$ and $\left.f_{2}^{\sigma}\right)$ values for gemini-conventional mixed surfactant systems at $30^{\circ} \mathrm{C}$

\begin{tabular}{lccccc}
\hline Surfactant System & $X_{1}^{\sigma}$ & $\beta^{\sigma}$ & $f_{1}{ }^{\sigma}$ & $f_{2}{ }^{\sigma}$ & $\begin{array}{c}\Delta \mathrm{G}_{\mathrm{ex}} \\
(\mathbf{K J} / \mathbf{m o l})\end{array}$ \\
\hline G5-CTAB & 0.5 & 8.00757 & 7.403055 & 7.403055 & 5.0430522 \\
G5-Brij35 & 0.5 & 5.05313 & 3.53701 & 3.53701 & 3.1823898 \\
G5-SDS & 0.5 & 11.3143 & 16.92126 & 16.92126 & 7.1255729 \\
\hline
\end{tabular}




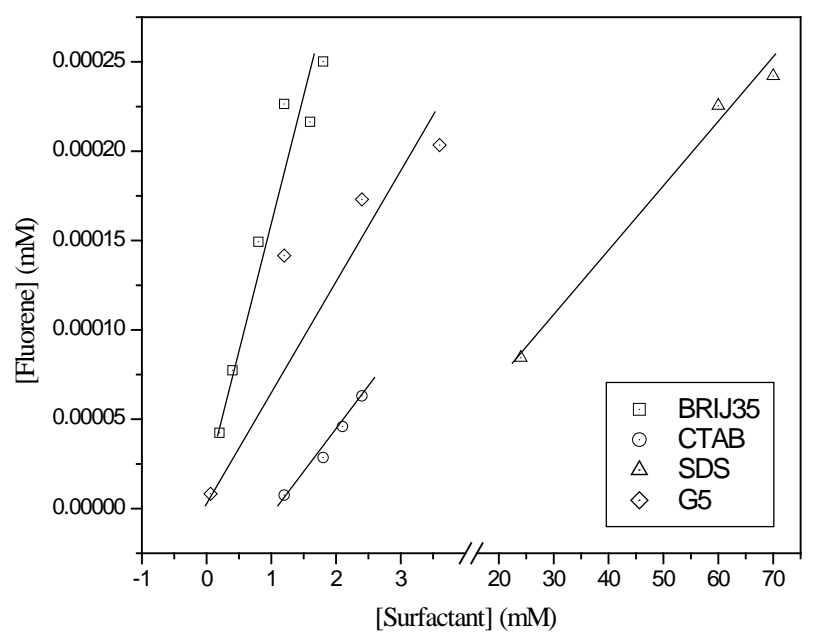

Figure 4. Variation of solubility of FLR with surfactant concentration.

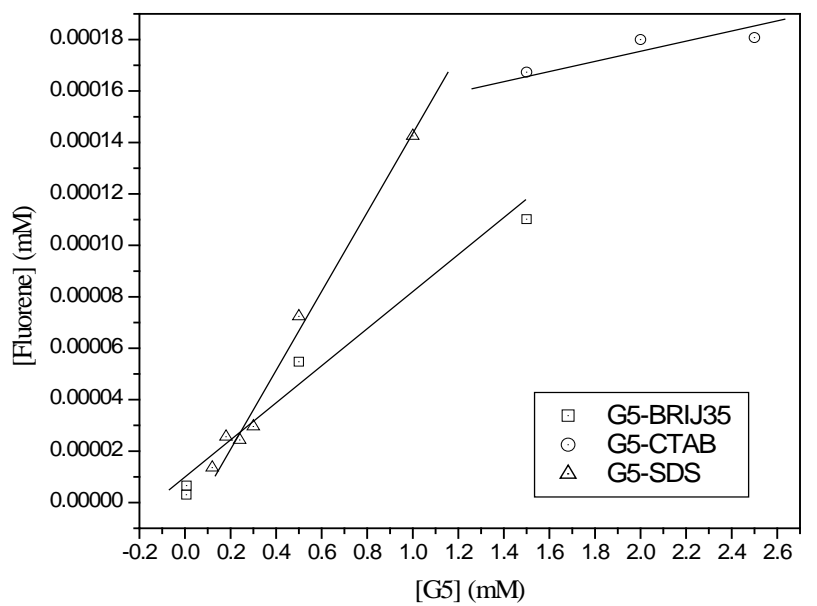

Figure 5. Variation of solubility of Fluorene with G5 concentration in 1:1 binary surfactant.

A measure of the effectiveness of a surfactant in solubilizing a given solubilizate is the Molar Solubilization Ratio, (MSR) which is given by [25, 28-33].

$$
\text { MSR }=\left\{\left[\mathrm{S}_{\mathrm{t}}\right]-\left[\mathrm{S}_{\mathrm{cmc}}\right]\right\} /\left\{\mathrm{C}_{\mathrm{t}}-\mathrm{CMC}\right\}
$$

where $S_{c m c}$ and $\mathrm{S}_{\mathrm{t}}$ are the solubilities at CMC and at total surfactant concentration $C_{t}$ respectively. Since $\left(\mathrm{C}_{t}-\mathrm{CMC}\right)$ was the concentration of the surfactant in the micellar form, MSR was equal to the ratio of solubilizate concentration in the micelles to the concentration of surfactant in the form of micelles. Value of MSR is obtained from the slope of solubilizate concentration versus surfactant concentration plot.

In the presence of excess FLR, MSR values of both single and mixed surfactants can be obtained from the slope of the linearly fitted line in which the concentration was plotted against surfactant concentration above the CMC (both the concentrations were in $\mathrm{mM}$ ) given in Figures 4-5. One can obtain the fact that as on increasing surfactant concentration, FLR concentration is also risen up, which provides positive value of MSR. The effectiveness of solubilization can also be expressed with the help of the partition coefficient $K_{m}$ [17,32] which is defined as distribution of the mole fraction of FLR between surfactant micelles and the aqueous phase. It may be calculated as [32]:

$$
\mathrm{K}_{\mathrm{m}}=\mathrm{X}_{\mathrm{m}} / \mathrm{X}_{\mathrm{a}}
$$

where $X_{m}$ and $X_{a}$ are the mole fraction of FLR in micelle phase and mole fraction of FLR in aqueous phase. The quantity $\mathrm{X}_{\mathrm{m}}$ can be expressed in terms of MSR, as

$$
\mathrm{X}_{\mathrm{m}}=\frac{\text { MSR }}{(1+\mathrm{MSR})}
$$

Mole fraction of the solute in the aqueous phase was approximated for dilute solution by:

$$
\mathrm{X}_{\mathrm{a}}=\mathrm{S}_{\mathrm{CMC}} \mathrm{V}_{\mathrm{W}}
$$

where $S_{\text {СMC }}$ is the total apparent solubility of the solute at $\mathrm{CMC}$ and $\mathrm{V}_{\mathrm{W}}$ is the molar volume of water $\left(1.807 * 10^{-2}\right.$ $\mathrm{L} / \mathrm{mol}$ at $30^{\circ} \mathrm{C}$ ). So, the $\mathrm{K}_{\mathrm{m}}$ expression can be rearranged as:

$$
\mathrm{K}_{\mathrm{m}}=\frac{\text { MSR }}{(1+\mathrm{MSR}) \mathrm{V}_{\mathrm{W}} \mathrm{S}_{\mathrm{CMC}}}
$$

As observed from Table 4 the MSR and $K_{m}$ values were highest for cationic surfactant and lowest for anionic and follow the order as Brij35 > G5 > CTAB > SDS. The order of solubilizing power for organic solutes by inner nonpolar core of micelles has been reported to be nonionic $>$ cationic $>$ anionic surfactant having same nonpolar chain length [24, 31-32]. For the case of FLR our observed data support these findings. The difference in solubilization capabilities of surfactant is because of their different structures. Higher solubilization power of Brij35 than G5 and SDS may be due to its larger micellar size helping in more micellar core solubilization [22]. The cationic surfactant exhibited lower MSR than nonionic due to limited solubilization at micelle/water interface and core of micelle.

\subsection{Solubilization by Equimolar Binary Mixed Surfactant Systems}

When MSR values were compared for all the mixed systems, the order was found as: G5-SDS > G5- Brij35 > G5-CTAB. MSR and $\log \mathrm{K}_{\mathrm{m}}$ values of cationic-nonionic surfactants were found higher than for cationic-cationic mixed surfactant solutions as previously reported by Wei et al. [30].

This is because nonionic micelle processing higher micellar core solubilization characteristic interpolates to the micelle-water interface producing greater solubilization power towards PAHs. In addition, the solubilization 
Table 5. Molar solubilization ratio (MSR), $\log K_{m}$, the free energy of solubilization $\left(\Delta G_{s}^{0}\right)$, $R$, and, B values for FLR solubilized in individual and mixed surfactant systems at $30^{\circ} \mathrm{C}$.

\begin{tabular}{lccccc}
\hline Surfactant System & MSR * $\mathbf{1 0}^{\mathbf{5}}$ & $\mathbf{L o g} \mathbf{K}_{\mathrm{m}}$ & $\mathbf{A G}_{\mathbf{s}}{ }^{0}(\mathbf{K J} / \mathbf{m o l})$ & $\mathbf{R}$ & $\mathbf{B}$ \\
\hline CTAB & 13.8 & 2.8539 & -16.56 & - & - \\
Brij35 & 38.2 & 3.2965 & -19.13 & - & - \\
SDS & 1.07 & 1.7423 & -10.11 & - & - \\
G5 & 15.6 & 2.9064 & -16.86 & 0.13 & -5.46 \\
G5-CTAB & 2.01 & 2.0174 & -11.70 & 0.39 & -1.75 \\
G5-Brij35 & 10.57 & 2.7384 & -15.89 & 2.17 & 12.25 \\
G5-SDS & 22.44 & 3.0652 & -17.78 & & - \\
\hline
\end{tabular}

of PHE and FLR by mixed gemini/nonionic surfactant solutions is also higher than those in single nonionic surfactant. In the interest of ascertaining the mixing effect of mixed surfactants on solubilization for PAHs and seeing the nature of deviation, the deviation ratio $(\mathrm{R})$ between the $\mathrm{MSR}_{\text {exp }}$ and the $\mathrm{MSR}_{\text {ideal }}$ can be determined by the following equation:

$$
R=\frac{\mathrm{MSR}_{\text {exp }}}{\mathrm{MSR}_{\text {ideal }}}
$$

$\mathrm{MSR}_{\text {ideal }}$ is the MSR for organic compounds in mixed surfactant system at the ideal mixed state and can be estimated using the MSR of single surfactant solutions based on the ideal mixing rule:

$$
\mathrm{MSR}_{\text {ideal }}=\mathrm{MSR}_{1} \mathrm{X}_{1}+\mathrm{MSR}_{2} \mathrm{X}_{2}
$$

where $\mathrm{X}_{1}, \mathrm{X}_{2}, \mathrm{MSR}_{1}$ and $\mathrm{MSR}_{2}$ are the mole fraction and the molar solubilization ratio for solute of components 1 and 2 in mixed surfactant solutions, respectively. The data of parameter $\mathrm{R}$ from Table 5 obviously indicate that the $\mathrm{MSR}_{\exp }$ values have positive deviation from ideal mixture for the gemini/nonionic and gemini/anionic surfactant systems meaning that they have positive mixing effect on solubilization of FLR. Another parameter $\mathrm{K}_{\mathrm{m} 12}$, the partition coefficient of a neutral organic solute between micelles and aqueous phase in a mixed surfactant has been used by Treiner et al. [32]. This parameter provides better understanding of the mixing effect of mixed surfactant systems on solubilization of solutes.

This partition coefficient's expression is based on the regular solution approximation as follows:

$$
\begin{aligned}
\ln \mathrm{K}_{\mathrm{m} 12}= & \mathrm{X}_{1}^{\mathrm{m}} \ln \mathrm{K}_{\mathrm{m} 1}+\left(1-\mathrm{X}_{1}^{\mathrm{m}}\right) \ln \mathrm{K}_{\mathrm{m} 2} \\
& +\mathrm{B} \mathrm{X}_{1}^{\mathrm{m}}\left(1-\mathrm{X}_{1}^{\mathrm{m}}\right)
\end{aligned}
$$

where $\mathrm{K}_{\mathrm{m} 1}, \mathrm{~K}_{\mathrm{m} 2}$ are the micelle-water partition coefficients of individual surfactant solutes constituting the mixed micelles and $\mathrm{X}_{1}^{\mathrm{m}}$ represents the micellar mole fraction of a surfactant having the value of $K_{m 1}$. B is an empirical parameter involving both the surfactantsurfactant and surfactant-solute interactions. If value of $\mathrm{B}$ becomes 0 it means there would be no mixing effect on partitioning of a solute between aqueous and micellar phase. Whereas for $\mathrm{B}>0(<0)$ implies that $\mathrm{K}_{\mathrm{m} 12}$ in the mixed surfactant system is larger (smaller) than predicted by ideal mixing rule. As presented in Table 5, the B values are found to be negative for all the equimolar binary mixed systems.

\section{Conclusions}

The experimental results obtained in the present study may be useful for the selection of appropriate mixed surfactant systems. This study would also facilitate the design and optimization of new surfactant systems for their better performance. The mixed micelles of gemini surfactant G5 with all the conventional surfactants i.e. Brij35, CTAB and SDS are studied. Highest attracting interaction in mixed micelle formation is observed in G5-SDS and lowest attracting interaction G5-Brij35, which indicates good synergism in mixed micelles. $\Delta G_{m}$ and $\Delta G_{a d}$ values are negative in all systems and show the spontaneity. The values of $\Delta G_{e x}$ are negative for all mixed systems, demonstrating the stability of the micelles.

\section{REFERENCES}

[1] Y. Zhang, W. J. Maier and R. M. Miller, Environmental Science Technology, Vol. 31, No. 8, 1997, pp. 2211-2217.doi:10.1021/es960687g 
[2] S. Laha and R. G. Guthy, Environmental Science Technology, Vol. 25, 1991.

[3] M. B. Gu and S. K. Chang, "Biosensors \& Bioelectronics,” Vol. 16, No. 9-12, 2001, pp. 667-674. doi:10.1016/S0956-5663(01)00230-5

[4] A. Mohamed and S. M. Mahfoodh, "Colloids Surfaces A: Physicochemical and Engineering Aspects,” Vol. 287, No. 1-3, 2005, pp. 44-50. doi:10.1016/j.colsurfa.2006.03.036

[5] L. A. Bernardez and S. Ghoshal, "Solubilization Kinetics for Polycyclic Aromatic Hydrocarbons Transferring from a Non-aqueous Phase Liquid to Non-ionic Surfactant Solutions, Journal of Colloid Interface Science, Vol. 320, 2008, pp. 298-306. doi:10.1016/j.jcis.2007.12.035

[6] J. C. Dur, R. Rama, H. Parola and V. Chaplin, Heterogeneous dissolution of benzo(a)pyrene by surfactant solutions, Colloids and Surfaces A: Physicochemical and Engineering Aspects, Vol. 162, No. 1-3, 1999, pp. 249-257. doi:10.1016/S0927-7757(99)00244-7

[7] D. Grasso, K. Subramaniam, J. J. Pignatello, Y. Yang and D. Ratte, Colloids and Surfaces A: Physicochemical and Engineering Aspects, Vol. 194, 2001, pp. 65-74.doi:10.1016/S0927-7757(01)00800-7

[8] C. K. Ahn, S. H. Woo and J. M. Park, "Selective adsorption of phenanthrene in nonionic-anionic surfactant," Chemical Engineering Journal, Vol. 158, No. 2, 2010, pp. 115-119. doi:10.1016/j.cej.2009.12.014

[9] M. Panda and Kabir-ud-din, J. Mol. Liq., Vol. 163, No. 2, 2001, pp. 93-98. doi:10.1016/j.molliq.2011.08.002

[10] M. Almgren, V. M. Garamus, L. Nordstierna, J. Luc-Blin and M. J. Stebe, Langmuir, Vol. 29, No. 36, 5355 (2010). doi:10.1021/la903764u

[11] G. B. Ray, S.Ghosh and S. P. Moulik, J. Chem. Sci., 122, 109 (2010). doi:10.1007/s12039-010-0011-1

[12] D. Tikariha, K. K. Ghosh, N. Barbero, P. Quagliotto and S. Ghosh, Colloids and Surfaces A: Physicochemical and Engineering Aspects, Vol. 381, No. 1-3, 2011, pp. 61-69. doi:10.1016/j.colsurfa.2011.03.027

[13] J. R. Akbar, R. Deubry, D. G. Marangoni and S. D. Wettig, J. Mol. Liq., 88, 321 (2010).

[14] F. M. Menger and C. A. J. Littau, "Gemini-surfactants: synthesis and properties," Journal of the American chemical society, Vol. 113, No. 4, 1991, pp. 1451-1452. doi:10.1021/ja00004a077

[15] B. S. Sekhon, Resonance, 42, (2004).

[16] K. S. Sharma, C. Rodgers, R. M. Palepu and A. K. Rakshit, Studies of Mixed Surfactant Solutions of Cationic Dimeric (Gemini) Surfactant with Nonionic Surfactant $\mathrm{C}_{12} \mathrm{E}_{6}$ in Aqueous Medium," Journal of Colloid Interface Science, Vol. 268, No. 2, 2003, pp. 482-488. doi:10.1016/j.jcis.2003.07.038

[17] S. P. Moulik and S. Ghosh, "Surface Chemical and Micellization Behaviours of Binary and Ternary Mixtures of Amphiphiles (Triton X-100, Tween-80 and CTAB) in Aqueous Medium,” Journal of Molecular Liquids, Vol.
72, No. 1-3, 1997, pp. 145-161. doi.org/10.1016/S0167-7322(97)00036-6

[18] J. H. Clint, J. Chem. Soc Faraday Trans., Vol. 71, 1974, p. 1327.

[19] T. Joshi., J. Mata and P. Bahadur, "Micellization and Interaction of Anionic and Nonionic Mixed Surfactant Systems in Water," Colloids and Surfaces A: Physicochemical and Engineering Aspects, 260, 209 (2005). doi.org/10.1016/j.colsurfa.2005.03.009

[20] D. N. Rubnigh, in: K. L. Mittal (Ed.)Solution Chemistry of Surfactants. Vol. 1, 1979, pp. 337-354. doi:10.1007/978-1-4615-7880-2_15

[21] K.J. Rao and S. Paria, Solubilization of Naphthalene in the Presence of PlantSynthetic Mixed Surfactant Systems,” Journal of Physical Chemistry B, Vol. 113, 2009, p. 474.doi:10.1021/jp8071298

[22] M. J. Rosen, Surfactant and Interfacial Phenomena. Third ed., John Wiley \& sons Inc .Publication, 2004.

[23] M. Almgren, F. Grieser, J. K. Thoms, Journal of the American Chemical Society, Vol. 101, 1979, p. 279. doi:10.1021/ja00496a001

[24] J.L- Li, B.H. Chen, Chem. Eng. Sci., Vol. 118, 2002, p. 2825.

[25] L. Zhu, Chemosphere, Vol. 53, 2003, p. 459. doi:10.1016/S0045-6535(03)00541-1

[26] O. Zheng, "Solubilization of Pyrene in Aqueous Micellar Solutions of Gemini Surfactants C12-s-C12 2B r," Journal of Colloid Interface Science, Vol. 300, No. 2, 2006, pp. 749-754.doi:10.1016/j.jcis.2006.04.033v

[27] S. Paria, and P. K. Yuet, J. Am. Chem. Soc., Vol. 45, p. 3552, 2006.

[28] D. A. Edwards, R. G. Luithy and Z. Liu, "Solubilization of Polycyclic Aromatic Hydrocarbons in Micellar Nonionic Surfactant Solutions,” Environmental Science Technol, Vol. 25, No. 1, 1991, pp. 127-133. doi:10.1021/es00013a014

[29] S. Saito, "Solubilization Properties of Polymer-surfactant Complexes," Journal of Colloid and Interface Science, Vol. 24, No. 2, 1967, pp. 227-234. doi:10.1016/0021-9797(67)90225-1

[30] J. Wei, G. Haung and C. An, “Efficiency of Single and Mixed Gemini/conventional Micelles on Solubilization of Phenanthrene," Journal of Chemical Engineering, Vol. 168, No. 1, 2011, pp. 201-207. doi:10.1016/j.cej.2010.12.063

[31] W. Zhou and L. Zhu, "Colloids and Surfaces A: Physicochemical and Engineering Aspects," Colloids Surf. A: Physicochem and Engineering Aspects, Vol. 255, No. 1-3, 2005, pp. 145-152.doi:10.1016/j.colsurfa.2004.12.039

[32] C. Triener, M. Nortz and C. Vaution, Langmuir, Vol. 6, 1990, p. 127.

[33] W. J. Zhou and L. Z. Zhu, "Solubilization of pyrene by anionic-nonionic mixed surfactants," Journal of Hazardous Materials, Vol. 109, No. 1-3, 2004, pp. 213-220. doi:10.1016/j.jhazmat.2004.03.018 